\title{
Dynamic testing: new insights with polarization-sensitive optical coherence tomography in the Fourier domain
}

\author{
E. Leiss-Holzinger ${ }^{1}$, B. Heise ${ }^{1,2}$, B. Baumann ${ }^{3}$, E. Götzinger ${ }^{3}$, M. Pircher $^{3}$, C.K. Hitzenberger ${ }^{3}$, \\ D. Stifter ${ }^{4 a}$ \\ ${ }^{1}$ Recendt GmbH, 4020 Linz, Austria \\ ${ }^{2}$ FLLL - Department of Knowledge-based Mathematical Systems, JK University Linz, Austria \\ ${ }^{3}$ Center for Biomedical Engineering and Physics, Medical University of Vienna, Austria \\ ${ }^{4}$ ZONA - Center for Surface and Nanoanalytics, Johannes Kepler University Linz, Austria
}

\section{Introduction}

Optical coherence tomography (OCT), originally developed for depth resolved imaging on the micron scale within turbid tissue for biomedical diagnostics [1], is gaining increasing interest for alternative applications, like in the field of non-destructive testing and evaluation [2]. As an example, we have recently shown that polarization-sensitive OCT (PS-OCT) is capable of mapping the internal strain state in semitransparent materials in a contact-free way [3], as demonstrated on stationary samples. The promising concept of spectral-domain OCT (SD-OCT), i.e. a variant of Fourier-domain OCT with high sensitivity and imaging speed, is now exploited - for the first time to the best of our knowledge - to image the strain distribution of samples subjected to dynamic loading.

\section{Experimental}

The experiments were performed with a custom-built SD-PS-OCT system operating at $1.55 \mu \mathrm{m}$ imaging wavelength for increased imaging depth within scattering samples, as compared to the usually used $800 \mathrm{~nm}$ wavelength range [2]. A sketch of the setup is depicted in figure 1.

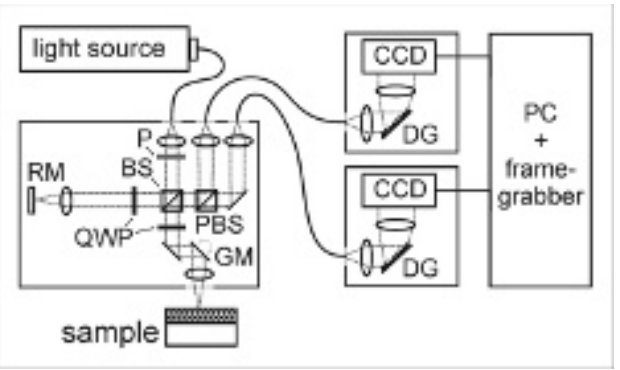

Fig. 1. Sketch of SD-PS-OCT setup. Reference mirror (RM), polarizer (P), beamsplitter (BS), polarizing BS (PBS), quarter wave plate (QWP), galvo scanner mirror (GM), diffraction grating (DG), line camera (CCD)

\footnotetext{
a e-mail : david.stifter@jku.at
} 


\section{Results and Discussion}

Fibre composite materials as well as injection moulded polymer parts were imaged with our new SD-PS-OCT system during dynamic loading and fracture tests, as depicted in the example of figure 2 (selected images taken from a movie). The imaging light is impinging from top on a fibre reinforced epoxy sheet (thickness $\sim 500 \mu \mathrm{m}$ ), which is bent over a rigid edge until fracture occurs. The back-reflected and back-scattered light is collected and evaluated to give the internal structure exhibiting individual fibre bundles (top row of figure 2). The arrow in the middle image indicates a crack running between two fibre bundles. The arrows in the right image show small parts of debris flying off the sample surface during fracture. In addition to the internal geometrical structure, the internal strain state can be mapped by evaluating the optical retardation of the principal polarization directions of the reflected light, as depicted in the bottom row. The bright regions (marked by the dotted ellipse in the middle image) indicate regions of high strain.

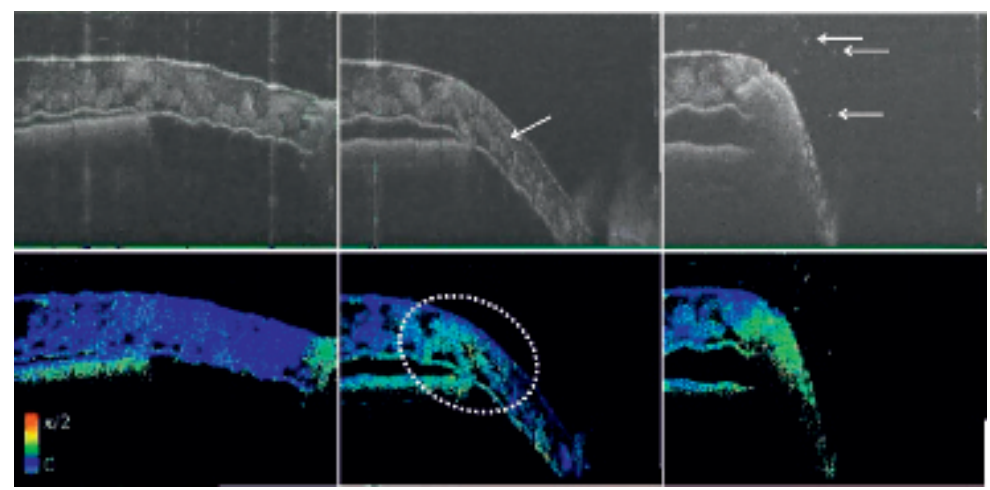

Fig. 2. SD-PS-OCT cross-sectional images of a fibre composite acquired during a fracture test.

Furthermore, advanced image processing techniques - applying coherent enhanced diffusion methods in conjunction with 2D-unwrapping algorithms - are used to quantitatively evaluate the internal stress distribution, as we will present for cyclic loading tests of polymer specimens with arbitrary shape.

Summarizing, SD-PS-OCT will be shown to be a potent technique which gives, in contrast to conventional photoelasticity methods, detailed and time-resolved information on the internal distribution of sample features, defects, stress and stress orientation.

\section{Acknowledgments}

This work was financially supported by the Austrian Science Fund (FWF), project P19751-N20, by the European Regional Development Fund (EFRE), and the federal state Upper Austria.

\section{References}

[1] D. Huang, E. A. Swanson, C. P. Lin, J. S. Schuhman, W. G. Stinson, W. Chang, M. R. Hee, T. Flotte, K. Gregory, C. A. Puliafito, J. G. Fujimoto, Science 254, 1178 (1991)

[2] D. Stifter, Appl. Phys. B 88337 (2007)

[3] B. Heise, K. Wiesauer, E. Götzinger, M. Pircher, C.K. Hitzenberger, R. Engelke, G. Ahrens, G. Grützner, D. Stifter, Strain (early view), doi: 10.1111/j.1475-1305.2008.00589.x (2008). 